\title{
An application of the multilayer perceptron: Solar radiation maps in Spain
}

\author{
L. Hontoria ，J. Aguilera , P. Zufiria
}

\begin{abstract}
In this work an application of a methodology to obtain solar radiation maps is presented. This methodology is based on a neural network system [Lippmann, R.P., 1987. An introduction to computing with neural nets. IEEE ASSP Magazine, 4-22] called Multi-Layer Perceptron (MLP) [Haykin, S., 1994. Neural Networks. A Comprehensive Foundation. Macmillan Publishing Company; Hornik, K., Stinchcombe, M., White, H., 1989. Multilayer feedforward networks are universal approximators. Neural Networks, 2(5), 359-366]. To obtain a solar radiation map it is necessary to know the solar radiation of many points spread wide across the zone of the map where it is going to be drawn. For most of the locations all over the world the records of these data (solar radiation in whatever scale, daily or hourly values) are nonexistent. Only very few locations have the privilege of having good meteorological stations where records of solar radiation have being registered. But even in those locations with historical records of solar data, the quality of these solar series is not as good as it should be for most purposes. In addition, to draw solar radiation maps the number of points on the maps (real sites) that it is necessary to work with makes this problem difficult to solve. Nevertheless, with the application of the methodology proposed in this paper, this problem has been solved and solar radiation maps have been obtained for a small region of Spain: Jaén province, a southern province of Spain between parallels $38^{\circ} 25^{\prime} \mathrm{N}$ and $37^{\circ} 25^{\prime} \mathrm{N}$, and meridians $4^{\circ} 10^{\prime} \mathrm{W}$ and $2^{\circ} 10^{\prime} \mathrm{W}$, and for a larger region: Andalucía, the most southern region of Spain situated between parallels $38^{\circ} 40^{\prime} \mathrm{N}$ and $36^{\circ} 00^{\prime} \mathrm{N}$, and meridians $7^{\circ} 30^{\prime} \mathrm{W}$ and $1^{\circ} 40^{\prime} \mathrm{W}$.
\end{abstract}

Keywords: Solar radiation; Neural networks; Solar radiation maps

\section{Introduction}

The availability of information about the solar radiation in the zone where a solar system is going to be installed is a necessary data for the designer of solar systems. This information, in case that it exists, can be 


\section{Nomenclature}

$\begin{array}{llll}d_{n} & \text { days } & k_{t} & \text { hourly clearness index } \\ h_{n} & \text { hour order number } & \text { MRV } & \text { mean relative variance }\end{array}$

$K_{T} \quad$ daily clearness index

available, in several ways. The most common is in tabular form with a lot of very useful information, usually large solar radiation sequences, but extremely difficult to handle. Another way can be solar radiation maps of the zone where the installation is going to be made. This second way is usually more efficient, easy to handle and preferable by the designers, to be used in a first approach in the solar system design and sizing.

Many procedures have been developed to draw solar radiation maps. In most of them, to obtain a solar radiation map it is necessary to know the solar radiation of many points spread wide across the real zone of the map where it is going to be drawn. These solar data can be available in several time scales. Usually there are two time scales in which the data are available: daily and hourly scales.

Nevertheless, for most of the locations all over the world the records of solar radiation data are non-existent. Only very few locations have the privilege of having good meteorological stations where records of solar radiation have being registered. Even in the locations with historical records of solar data, the quality of these solar series is not so good (Gansler et al., 1994).

So the problem of obtaining a solar radiation map in a certain zone is difficult to solve, due to the fact that it is necessary to know the solar radiation of a great number of sites. In this work a methodology to obtain solar radiation maps is presented. The methodology proposed makes use of a neural network system called MultiLayer Perceptron (MLP) (Haykin, 1994; Hornik et al., 1989; Lippmann, 1987). This neural network has already been presented in previous works (Hontoria et al., 1999, 2000, 2001, 2002; Zufiria et al., 1999). In those works the capabilities of the MLP to generate synthetic hourly solar radiation series have been demonstrated. Moreover, in comparison with other methods for generating synthetic sequences of solar data, the MLP improves (Hontoria et al., 1999, 2001) the results obtained by those methods.

In this case, an improvement of the neural system has been made, with the objective of the application of this neural supervised model to draw solar radiation maps. This methodology, as will be seen, is easily extendable and applicable to any other region of the world.

The article is divided in to 7 sections. Section 1 is this introduction. In Section 2 a summary of recurrent neural networks and time series prediction is done. In Sec- tion 3 the methodology to obtain solar radiation maps is introduced. Section 4 describes the universality capabilities of multilayer perceptron. Section 5 deals with the MLP architecture proposed. The solar radiation maps are presented in Section 6. Finally the conclusions and future actions are presented in Section 7.

\section{Recurrent neural networks and time-series generation}

\subsection{Artificial neural networks}

In the last fifteen years, artificial neural networks have been extensively employed in numerous fields of science and technology (Agarwal, 1997; Haykin, 1994; Hush and Horne, 1993; Kohonen, 1995; Lippmann, 1987). As a computation and learning paradigm, they are being proposed as an alternative approach for addressing complex problems. Among the many types of networks, supervised models have consolidated as the most robust and easy to employ when applicable (Hush and Horne, 1993). Usually, these models are implemented via feedforward architectures such as, for instance, the Multi-Layer Perceptron (MLP). One of the most appealing properties of these neural network architectures is their potential use for functional approximation, due to their universal approximation capabilities (Barron, 1993; Cybenko, 1989; Funahashi, 1989; Hornik et al., 1989).

Nevertheless, although the existence of architectures (with corresponding weight values) providing arbitrary small approximation errors is guaranteed, their efficient determination via some training procedure is still an open challenge. In addition, there are many problems where the availability of data and the possibility of their use for performing a supervised training process are not obvious. This is the case when dynamic or time-varying information is available.

\subsection{Multilayer perceptron based time series prediction}

The Multi-Layer Perceptron (MLP) is the most widely used type of supervised neural network for approximation tasks, its topology defining several layers of neurones. The MLP, in static contexts, is usually trained via a simple gradient-descent-based supervised procedure. The specific topology of the MLP makes 
the application of the gradient-descent method a very efficient process called the backpropagation algorithm (Rumelhart and MacClelland, 1986; Werbos, 1974).

Also, in dynamic contexts, the identification and control of non-linear plants, as well as the time series prediction have been successfully addressed via feedback architectures of supervised neural models (Narendra and Parthasarathy, 1990, 1991; Weigend et al., 1990; Zufiria, 1995). The basic TSP problem considers the availability of a time series $\left\{s_{n}\right\}$ up to an index value $N$; the objective is to predict future values of $\left\{s_{n}\right\}$ (i.e., for index values greater than $N$ ). The dynamical system approach to time series prediction assumes the existence of a function $G$ that relates each series value with the previous $p$ values

$s_{n+1}=G\left(s_{n-p+1}, \ldots, s_{n}\right), \quad p<n<N$

so that $\left\{s_{n}\right\}$ can be seen as a trajectory of the dynamical system (1). Assuming also that function $G$ can be computed, future values of the time-series could be estimated via

$\widehat{s}_{n+1}=G\left(s_{n-p+1}, \ldots, s_{n}\right), \quad n \geqslant N$

Hence, the problem reduces to the computation of function $G$. The MLP-based approach approximates such function via a MLP making use of the available data (time-series values up to index $N$ ). Therefore, the prediction procedure relies on the possibility of training the network so that

$\widehat{s}_{n+1}=G\left(s_{n-p+1}, \ldots, s_{n}\right) \cong \operatorname{MLP}\left(s_{n-p+1}, \ldots, s_{n}\right)$

By training a MLP with $p$ inputs and 1 output, with a training set representative enough, the MLP will be able to find the desired relationships between consecutive values (provided that they exist) just by approximating the function $G$. Once the approximation is performed, future values can be computed via feedback of the predictions whenever they are available. Such method is called prediction by network evolution (Zufiria, 1995). One of the great advantages of employing an MLP-based methodology for generating radiation series is that most of the computational resources are required during the training procedure, as opposed to the generation procedure.

\section{Proposed methodology to draw solar radiation maps}

The preparation of solar radiation maps requires the previous knowledge of a lot of information, which can be summarised with these steps:

Step 1. Making a grid of the zone that is going to be represented in the map. Each point of the grid corresponds to a certain real site that will have some particular features as its latitude, its longitude and its altitude over sea level, besides the solar radiation data.

Step 2. For each point of that grid knowledge of long sequences of solar radiation is needed.

Step 3. Once the series of solar radiation is available for the entire grid, it is necessary to obtain some particular statistical values to draw the maps. For instance, it is interesting to summarise all that information in 12 maps, one for each month of the year. So, in this case, it is necessary to obtain the mean monthly values of the solar radiation data.

A crucial problem is found in step 2: usually, in certain sites there is no solar data available. Particularly in Andalucía, the most southern region of Spain, where solar radiation maps are being prepared that problem exists. Only in the some cities of the region such as Almería, Cádiz, Córdoba, Huelva, Jaén, Málaga and Sevilla records of solar radiation are available. Also, for Jaén province, a small region of southern Spain, only in Jaén city solar radiation data have been recorded.

With all the data available and training a MLP it is possible to generate long sequences of solar radiation data which are statistically equivalent to the real series, as has been demonstrated in previous works (Hontoria et al., 1999, 2000, 2001, 2002; Zufiria et al., 1999). Nevertheless, for the generation of hourly solar radiation series in another location of the region in study two problems arise which were solved previously (Hontoria et al., 2000). These two problems are:

Problem 1. Generation of solar series in other places different from those where the data are available. It is what we call universality capabilities of neural networks, particularly the multilayer perceptron.

Problem 2. Inclusion in the MLP of another piece of information available like the latitude or the altitude over sea level. This information is important due to the fact that in many sites there is no information related to solar radiation, as for instance monthly mean solar radiation values, number of sunshine hours, number of cloudy days.

\section{Capabilities of perceptrons}

In order to demonstrate important capabilities of perceptrons, an exhaustive study was done, similar to the study done in previous works (Hontoria et al., 1999, 2000, 2001, 2002; Zufiria et al., 1999). Different locations, with different features where took. In Table 1 these sites, with some of their characteristics are shown. All of those sites are Spanish locations.

Seven different cities have been chosen. The election of those seven locations was done due to the different climate they have. The seven places are cities of Spain 
Table 1

Locations in study

\begin{tabular}{lllr}
\hline Location & Climate & Latitude & Altitude (m) \\
\hline Oviedo & Atlantic & $43.35^{\circ}$ & 348 \\
Logroño & Continental & $42.46^{\circ}$ & 372 \\
Madrid & Extreme Continental & $40.45^{\circ}$ & 664 \\
Palma de Mallorca & Island Mediterranean & $39.33^{\circ}$ & 32 \\
Badajoz & Attenuated Continental & $38.89^{\circ}$ & 185 \\
Murcia & Arid Mediterranean & $38.00^{\circ}$ & 69 \\
Málaga & Mediterranean & $36.66^{\circ}$ & 7 \\
\hline
\end{tabular}

with different climates. Also these seven locations have different altitudes upon sea level and their latitudes vary almost $7^{\circ}$, from $43.35^{\circ}$ to $36.66^{\circ}$. The hourly solar radiation data of these seven locations were recorded by the National Meteorological Institute of Spain. For all the locations we have eight completed recorded years of hourly solar radiation. The years recorded are from 1977 to 1984 in all those locations except for Logroño where we have from 1981 to 1984 (only four years). The quality of the data base was contrasted by the National Meteorological Institute of Spain, and all the data revised by the authors. With the hourly solar radiation data of these locations available, the process followed in this study is this one:

(a) First of all, seven different MLPs are trained for each city. The way of doing this training is described in previous works (Hontoria et al., 1999, 2000, 2001, 2002; Zufiria et al., 1999).

For our computational experiments, a set of hourly irradiation values $k_{t}$ measured in the locations shown in Table 1, and its corresponding daily values $K_{T}$ have been used. As a first approach, in order to evaluate the quality of a generated series, the first years were considered as a training set, and the last year was employed for testing the validity of the generated series, in each location.

The proposed method has been developed via a step by step inclusion of the available associated information. The great advantage of this MLP based methodology is that explicit knowledge of the relationship among all the information sources is not needed. Such information sources can be progressively incorporated in different steps upon the proposed method. The details of this step-by-step procedure can be found in (Zufiria, 1995). The final procedure, employing a MLP in a mixed feedback-feedforward configuration, is shown in Fig. 1.

As described in (Hontoria et al., 1999, 2000, 2001, 2002; Zufiria et al., 1999) the seven inputs are:

$d_{n}$ : distance (days) between the value to be generated and the day with maximum value in the $\left\{k_{t}\right\}$ annual

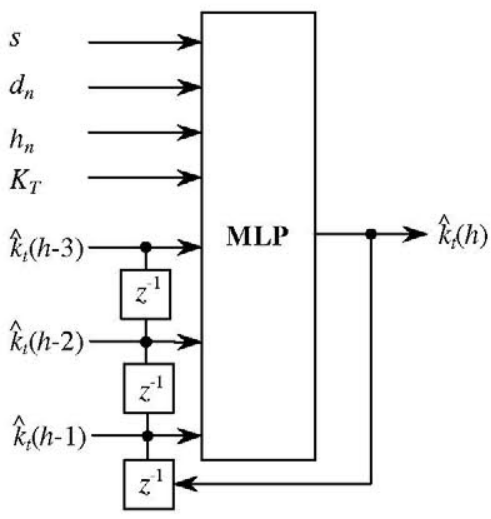

Fig. 1. MLP architecture for clearness indexes prediction.

distribution, in order to keep the monthly stationality,

$h_{n}$ : indicates the hour order number of the $k_{t}$ value (ensuring the hourly stationality). Both inputs $\left(d_{n}\right.$ and $h_{n}$ ) are normalised to the range $[0,1]$,

$K_{T}$ : daily clearness index,

$k_{t-1}, k_{t-2}, k_{t-3}$ : hourly clearness index of the three previous hours,

$s$ : this is an input taking the values $\{0,1\}$, that indicates whether an hour is between sunrise and sunset $\left(k_{t}\right.$ should be different from 0$)$ or not ( $k_{t}$ has to be 0$)$.

The training was done using the backpropagation algorithm (with momentum and random presentations), eventually combined with second order optimisation methods during the last few epochs. The hidden layer has fifteen nodes.

(b) Secondly, with the MLP trained in a location, hourly solar radiation data for the other six cities are generated with this MLP.

(c) Thirdly, new synthetic hourly solar radiation years are obtained by other classic generation methods. The classical methods employed are the Graham and Hollands method (GH method) (Graham and 
Hollands, 1990) and the Aguiar and Collares-Pereira method (AC method) (Aguiar and Collares-Pereira, 1992).

(d) Finally, with all of this information a comparative study is done. As a quality measure, the mean relative variance (MRV) (Hontoria et al., 1999) was used. This parameter, commonly employed in the digital signal processing community, quantifies the relative error, and it is defined as the quotient between the prediction error signal power and the AC power of the signal to be predicted

$$
\text { MRV }=\frac{\sum_{h}\left(k_{\mathrm{th}}-\hat{k}\right)^{2}}{\sum_{h}\left(k_{\mathrm{th}}-\bar{k}_{t}\right)^{2}}
$$

where $k_{\text {th }}^{\prime}$ are the values predicted, $k_{\text {th }}$ are the real values and $k_{t}^{-}$is the mean value of $k_{\mathrm{th}}$.

The results are shown in Fig. 2, where several MLPs have been tested. All of them were trained at the several locations in study. Afterwards, the generation of the solar data was done using the MLP trained in a particular location and using data of that location (what we called own MLP, in Fig. 2) or using MLP trained at other locations (as it can be seen in Table 2).

In Table 2, the number of the MLP indicates the place where it has been trained, for instance MLP1 in Oviedo, MLP2 Logroño, etc.

As can be seen the MRV values are better for the data obtained by the MLP than those obtained by the classical methods ( $\mathrm{GH}$ and $\mathrm{AC}$ ). Even for the data

MRV.

Real data against data generated by own MLP and methods AC and GH

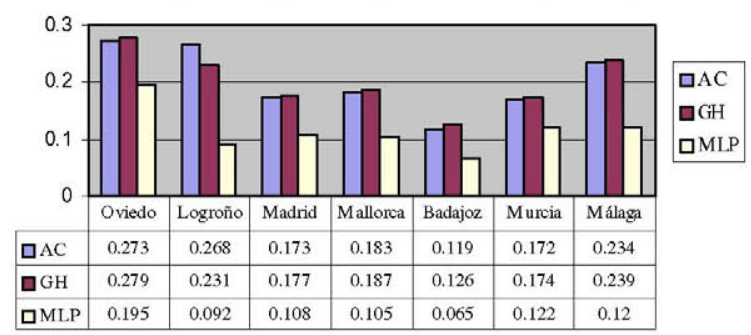

Fig. 2. (MRV) Real data against data generated by own MLP and the methods $\mathrm{AC}$ and $\mathrm{GH}$. obtained with the MLP trained with other cities data but applied in the other locations the MRV values are better than those of $\mathrm{GH}$ and $\mathrm{AC}$ method. With this study it can be concluded that the MLP presents important capabilities to generated solar radiation data, using data of places of similar latitude to the one the generation has being done.

\section{MLP Architecture proposed to draw solar radiation maps}

Once a MLP is trained in a certain site it can be used for the hourly solar generation in a different place. Nevertheless, for the hourly generation, some solar information in the location where the generation is taking place is needed. For instance, this solar information can be solar global radiation monthly mean values, number of sunshine hours, distribution of cloudy days, etc.

To draw solar maps in Andalucía the only available solar information was the hourly solar radiation data records in Almería, Cádiz, Córdoba, Huelva, Jaén, Málaga and Sevilla, the altitude upon sea level and the latitude. A MLP with this data was trained for those locations. Nevertheless, for the solar generation in some other sites of the region to obtain the solar maps different information was used. This new information is the latitude and altitude upon sea level. These two items were included in the MLP as new entrances.

A unique MLP was trained with the all the solar data available in Andalucía, modifying the structure of Fig. 1 with two new entrances corresponding to the latitudes and altitudes of these sites. This MLP is used for the hourly solar generation of each site of the grid in the solar maps. In the next section this procedure is described.

\section{Solar radiation maps}

\subsection{Solar radiation maps for small regions}

To obtain solar radiation maps we follow the steps mentioned in Section 3. For instance, to obtain solar radiation maps for Jaén province the procedure followed

Table 2

(MRV) Real data against data generated by different MLPs

\begin{tabular}{llllllll}
\hline Site & MLP1 & MLP2 & MLP3 & MLP4 & MLP5 & MLP6 & MLP7 \\
\hline Oviedo & 0.195 & 0.215 & 0.223 & 0.217 & 0.207 & 0.209 & 0.221 \\
Logroño & 0.158 & 0.092 & 0.145 & 0.151 & 0.139 & 0.147 & 0.139 \\
Madrid & 0.168 & 0.163 & 0.108 & 0.143 & 0.137 & 0.153 & 0.130 \\
Mallorca & 0.173 & 0.176 & 0.180 & 0.105 & 0.162 & 0.169 & 0.165 \\
Badajoz & 0.087 & 0.102 & 0.105 & 0.101 & 0.065 & 0.110 & 0.098 \\
Murcia & 0.167 & 0.146 & 0.162 & 0.157 & 0.152 & 0.122 & 0.137 \\
Málaga & 0.193 & 0.187 & 0.162 & 0.173 & 0.148 & 0.132 & 0.120 \\
\hline
\end{tabular}


is this one. Firstly, a grid with more than 250 points has been prepared. These points vary from parallels $38^{\circ} 25^{\prime}$ $\mathrm{N}$ to $37^{\circ} 25^{\prime} \mathrm{N}$, and meridians $4^{\circ} 10^{\prime} \mathrm{W}$ to $2^{\circ} 10^{\prime} \mathrm{W}$, and have been chosen in intervals of $5^{\prime}$. For all of this sites solar hourly values must be generated.

Secondly, as previously mentioned, a unique MLP has been trained. To train this MLP, hourly solar radiation data from Madrid, Oviedo, Málaga and Jaén have been used. In this case, the solar radiation data for the first three locations mentioned are the same as we described in Section 4, and for Jaén we have ten years of solar radiation data recorded by our researcher group. The election of the first three locations is due to the very different climates and latitudes they have. The choice of the location of Jaén is due to the fact that the maps are going to be made in its province. This MLP is the tool used for the generation of solar hourly values in all the sites of the grid of the map. The solar information used for the generation is the Jaén solar data, and the partic- ular information of each site is the latitude and altitude. So the idea is, using the solar data of Jaén and varying the values of the entrances "latitude" and "altitude" for each point on the grid, the MLP generates solar radiation data for every point on the grid.

Finally the solar global monthly mean values (Iqbal, 1983) are obtained for each point on the grid, so twelve solar radiation maps are possible to draw (one for each month). Next two figures (Figs. 3 and 4) present two maps obtained by this methodology. One map is for March (spring) and the other one is for September (autumn).

\subsection{Solar radiation maps for large regions}

The same procedure, but with some data variation has been done to obtain solar radiation maps of a large region. In this case the region in study is Andalucía. This is the most southern region of Spain. A grid with more

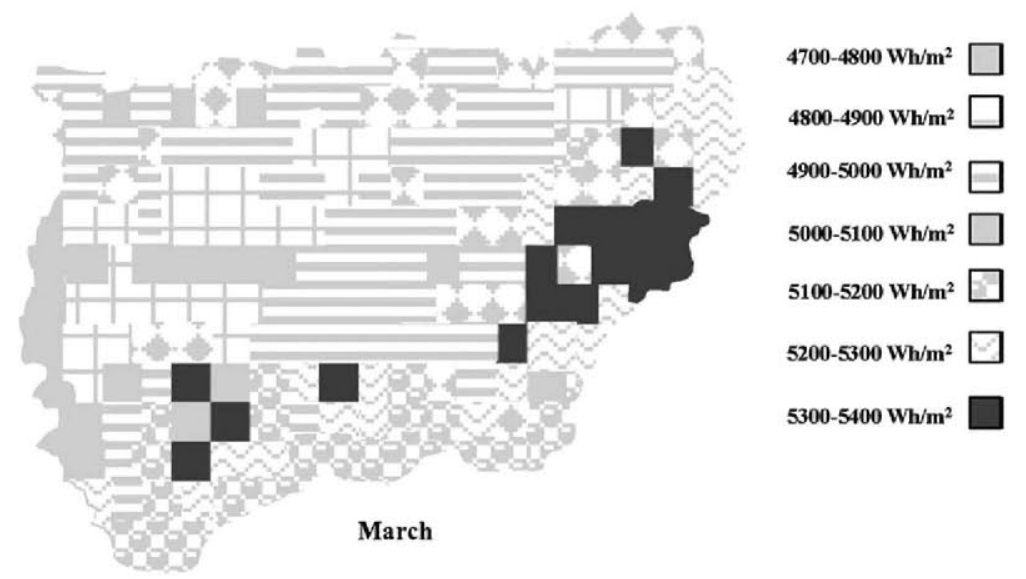

Fig. 3. Solar radiation map of Jaén province in March.
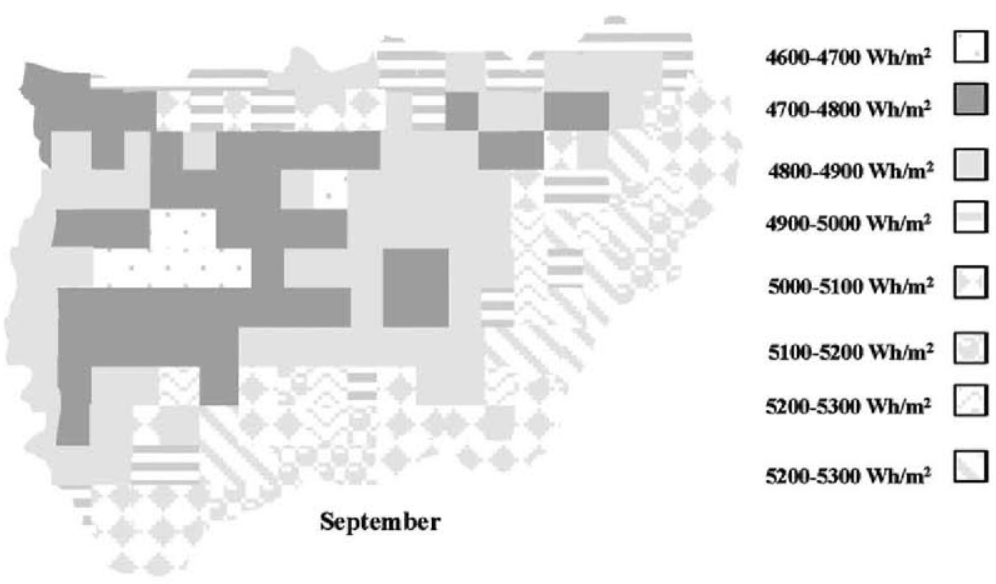

Fig. 4. Idem in September. 

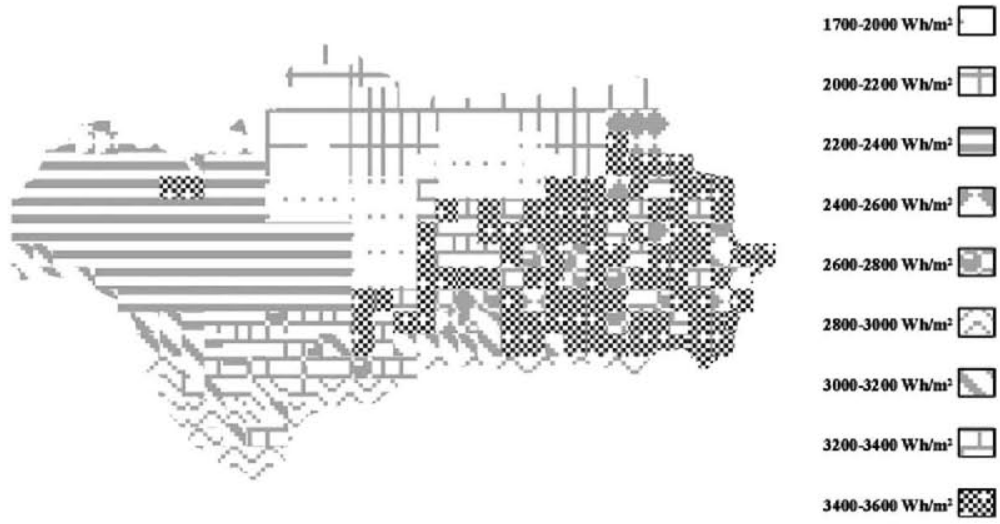

Fig. 5. Solar radiation map of Andalucia in December.
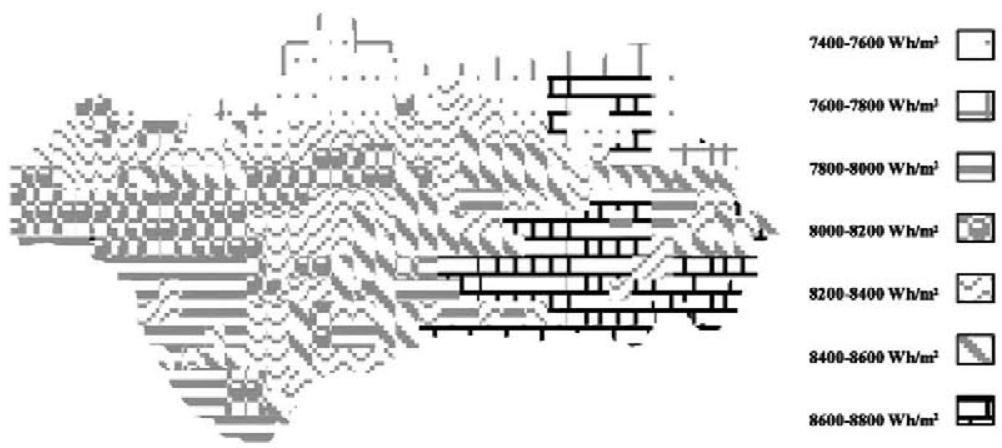

Fig. 6. Solar radiation map of Andalucía in June.

than 500 points has been prepared. These points vary from parallels $38^{\circ} 40^{\prime} \mathrm{N}$ and $36^{\circ} 00^{\prime} \mathrm{N}$, and meridians $7^{\circ} 30^{\prime} \mathrm{W}$ and $1^{\circ} 40^{\prime} \mathrm{W}$, and have been chosen in intervals of $10^{\prime}$. For all of these sites solar hourly values must be generated.

A MLP has been trained with hourly solar radiation data of sites in Andalucía. In this case, the solar radiation data available correspond to these locations: Jaén, Córdoba, Sevilla, Cádiz Huelva, Málaga, Granada and Almería. For those eight cities the National Meteorological Institute provides us with eight years of solar radiation. As in the previous section the solar global monthly mean values are obtained for each point on the grid, so twelve solar radiation maps were drawn (one for each month). Next two figures (Figs. 5 and 6 ) present two maps obtained by this methodology. One map is for December (winter) and the other one is for June (summer).

\section{Conclusions and future actions}

A new methodology for the obtaining of solar radiation maps has been developed. To obtain a solar radiation map of a certain zone it is necessary to know the solar radiation of a huge number of sites spread wide across the zone. This information is usually non-existent, so this problem has been solved with the methodology proposed.

The methodology proposed is based on a neural system called Multi-layer Perceptron (MLP). The MLP is a neural system that needs previous training and afterwards it can be applied in different places, varying only its entrances. The MLP proposed has been trained with hourly solar radiation data from seven sites in Spain. Each of these seven sites has different features such as climate, latitudes, and so on.

Once the MLP is trained a solar generation can be done in all of the sites of the grid, which form the zone map. This generation is extremely simple and takes less time than the same generation than classical methods of solar generation.

Besides, the classical methods are unable to generate solar radiation series in places where no solar information is available. Nevertheless, the methodology proposed is able to do this, it is more versatile than the classical methods, and so is able to draw maps of the zone.

This methodology is easily extendible to other places. The only requirement is the knowledge of the hourly solar radiation from only one site of the zone where the map is going to be drawn. 
As a first future action, maps of different provinces, regions or even countries will be prepared. The extension of this methodology to other areas is, as has been demonstrated quite easy. So drawing maps of more extended areas will be done.

It is very important to contrast artificial results with real data. Then a comparison with real data, in the places where they are available, will be done. Also the comparison will be done with other classical methods of solar radiation generation.

Finally it is necessary to do a statistically study of the generation data. In this study a lot of statistical parameters such as monthly mean values, variances, autocorrelations and so on will be taken into account.

\section{References}

Agarwal, M., 1997. A systematic classification of neuralnetwork-based control. IEEE Control Systems Magazine 17 (2), 75-93.

Aguiar, R., Collares-Pereira, M., 1992. TAG: a time-dependent, autoregressive, gaussian model for generating synthetic hourly radiation. Solar Energy 49 (3), 167-174.

Barron, A.R., 1993. Universal approximation bounds for superpositions of a sigmoidal function. IEEE Transactions Information Theory 39, 930-945.

Cybenko, G., 1989. Approximation by superpositions of a sigmoidal function. Mathematics of Control Signals and Systems 2, 303-314.

Funahashi, K., 1989. On the approximate realisation of continuous mappings by neural networks. Neural Networks 2, 183-192.

Gansler, R., Klein, S.A., Beckman, W.A., 1994. Assessment of the accuracy of generated meteorological data for use in solar energy simulation studies. Solar Energy 53 (3).

Graham, V.A., Hollands, G.T., 1990. A method to generate synthetic hourly solar radiation globally. Solar Energy 44 (6), 333-341.

Haykin, S., 1994. Neural Networks. A Comprehensive Foundation. Macmillan Publishing Company.

Hontoria, L., Riesco, J., Zufiria, P., Aguilera, J., 1999. Improved generation of hourly solar radiation artificial series using neural networks. EANN99. Varsovia.

Hontoria, L., Riesco, J., Zufiria, P., Aguilera, J., 2000. Application of neural networks in the solar radiation field. Obtainment of solar radiation maps. 16th European Pho- tovoltaic Solar Energy Conference and Exhibition. Glasgow, 1-5 May.

Hontoria, L., Aguilera, J., Riesco, J., Zufíria, P., 2001. Recurrent neural supervised models for generating solar radiation synthetic series. Journal of Intelligent and Robotic Systems 31, 201-221.

Hontoria, L., Aguilera, J., Zufíria, P., 2002. Generation of hourly irradiation synthetic series using the neural network multilayer perceptron. Solar Energy 72, 441-446.

Hornik, K., Stinchcombe, M., White, H., 1989. Multilayer feedforward networks are universal approximators. Neural Networks 2 (5), 359-366.

Hush, D.R., Horne, B.G., 1993. Progress in supervised neural networks. What's New Since Lippmann. IEEE S.P. Magazine, pp. 8-39, January.

Iqbal, M., 1983. An Introduction to Solar Radiation. Academic Press.

Kohonen, T., 1995. Self-Organising Maps. Springer-Verlag, Berlin Heidelberg.

Lippmann, R.P., 1987. An Introduction to Computing with Neural Nets. IEEE ASSP Magazine, pp. 4-22.

Narendra, K.S., Parthasarathy, K., 1990. Identification and control of dynamical systems using neural networks. IEEE Transactions on Neural Networks 1 (1), 4-27.

Narendra, K.S., Parthasarathy, K., 1991. Gradient methods for the optimisation of dynamical systems containing neural networks. IEEE Transactions on Neural Networks 2 (2), 252-262.

Rumelhart, D., MacClelland, J.L., 1986. Learning internal representations by error backpropagation. Parallel Distributed Processing, vol. 1. Foundations: The MIT Press (chapter 8).

Weigend, A.S., Rumelhart, D., Huberman, B.A., 1990. Backpropagation, weight-elimination and time series prediction. In: Proceedings of the 1990 Connectionist models Summer School. Morgan Kaufman.

Werbos, P., 1974. Beyond regression: new tools for prediction and analysis in the behavioural sciences. Ph.D. dissertation, Committee on Applied Mathematics, Harvard University, Cambridge, MA.

Zufiria, P., 1995. An overview of dynamic system control using neural networks. In: Bulsari, A.B. (Ed.), Neural Networks for Chemical Engineers. Elsevier, ISBN 0-444-82097-3, pp. 385-408.

Zufiria, P., Vázquez, A., Riesco, J., Aguilera, J., Hontoria, L., 1999. A neural network approach for generating solar radiation artificial series. In: Proceedings of the (IWANN'99). 4-5 June. Alicante, Spain. 